\title{
A Coaching by Telephone Intervention on Engaging Patients to Address Modifiable Cardiovascular Risk Factors: a Randomized Controlled Trial
}

\author{
Eugene Z. Oddone, MD, MHSc ${ }^{1,2}$, Jennifer M. Gierisch, PhD, MPH ${ }^{1,3}$, Linda L. Sanders, MPH' , \\ Angela Fagerlin, $P D^{4,5}$, Jordan Sparks, MPH' ${ }^{6}$, Felicia McCant, MSSW ${ }^{7}$, Carrie May, MPH', \\ Maren K. Olsen, $P h D^{1,7}$, and Laura J. Damschroder, $M P H, M S^{6}$
}

\begin{abstract}
${ }^{1}$ Center for Health Services Research in Primary Care, Durham VA Medical Center, Durham, NC, USA; ${ }^{2}$ Division of General Internal Medicine, Department of Medicine, Duke University Medical Center, Durham, NC, USA; ${ }^{3}$ Department of Population Health Sciences, Duke University Medical Center, Durham, NC, USA; “VA Salt Lake City Center for Informatics Decision Enhancement and Surveillance (IDEAS), Salt Lake City, UT, USA; ${ }^{5}$ Department of Population Health Sciences, University of Utah, Salt Lake City, UT, USA; ${ }^{6}$ VA Center for Clinical Management Research, VA Ann Arbor Healthcare System, Ann Arbor, MI, USA; ${ }^{7}$ Department of Biostatistics and Bioinformatics, Duke University, Durham, NC, USA.
\end{abstract}

BACKGROUND: A large proportion of deaths and chronic illnesses can be attributed to three modifiable risk factors: tobacco use, overweight/obesity, and physical inactivity. OBJECTIVE: To test whether telephone-based health coaching after completion of a comprehensive health risk assessment (HRA) increases patient activation and enrollment in a prevention program compared to HRA completion alone.

DESIGN: Two-arm randomized trial at three sites.

SETTING: Primary care clinics at Veterans Affairs facilities.

PARTICIPANTS: Four hundred seventeen veterans with at least one modifiable risk factor $(\mathrm{BMI} \geq 30,<150 \mathrm{~min}$ of at least moderate physically activity per week, or current smoker).

INTERVENTION: Participants completed an online HRA. Intervention participants received two telephonedelivered health coaching calls at 1 and 4 weeks to collaboratively set goals to enroll in, and attend structured prevention programs designed to reduce modifiable risk factors.

MEASUREMENTS: Primary outcome was enrollment in a structured prevention program by 6 months. Secondary outcomes were Patient Activation Measure (PAM) and Framingham Risk Score (FRS).

RESULTS: Most participants were male (85\%), white (50\%), with a mean age of 56. Participants were eligible, because their BMI was $\geq 30$ (80\%), they were physically inactive (50\%), and/or they were current smokers (39\%). When compared to HLA only at 6 months, health coaching intervention participants reported higher rates of enrollment in a prevention program, 51 vs $29 \%$ (OR $=2.5 ; 95 \%$ CI: $1.7,3.9 ; p<0.0001)$, higher rates of program participation, 40 vs $23 \%$ (OR = 2.3; 95\% CI: 1.5, 3.6; $p=0.0004)$, and greater improvement in PAM scores, mean difference 2.5 (95\% CI: 0.2, 4.7; $p=0.03$ ), but no change in FRS scores, mean difference 0.7 (95\% CI - 0.7, $2.2 ; p=0.33)$.

Received June 19, 2017

Revised November 17, 2017

Accepted March 1, 2018

Published online May 7, 2018
CONCLUSIONS: Brief telephone health coaching after completing an online HRA increased patient activation and increased enrollment in structured prevention programs to improve health behaviors.

CLINICALTRIALS.GOV IDENTIFIER: NCTO 1828567

KEY WORDS: telephone coaching; health risk assessment; patient engagement.

J Gen Intern Med 33(9):1487-94

DOI: $10.1007 / \mathrm{s} 11606-018-4398-6$

(c) Society of General Internal Medicine (outside the USA) 2018

$T$ hree modifiable risk factors, tobacco use, overweight/ obesity, and physical inactivity, are linked to over half of all deaths and many illnesses. ${ }^{1,2}$ The US population has a high prevalence of these risk factors and has the greatest number of preventable deaths among industrialized nations. ${ }^{3}$ Addressing these modifiable risk factors is particularly important for veterans, more than $70 \%$ of whom are overweight (body mass index $[\mathrm{BMI}] \geq 25 \mathrm{~kg} / \mathrm{m}^{2}$ ), $40 \%$ of whom are obese $\left(\mathrm{BMI} \geq 30 \mathrm{~kg} / \mathrm{m}^{2}\right)$, and $30 \%$ of whom smoke. ${ }^{4}$ Even worse, younger veterans are at particularly high risk for developing chronic illnesses, because they are more likely to be overweight/obese and smoke more heavily compared to nonveterans the same age. $^{5-7}$

A health risk assessment (HRA) is a comprehensive tool to elicit patient-reported behaviors and combine them with clinical values in a statistical model that presents to patients their risk for adverse outcomes, and the degree to which these outcomes can be modified by changing their behaviors. Comprehensive HRAs have been in use for over 30 years and are widely available online; however, HRA use alone is not effective in changing behaviors. ${ }^{8-10}$ Health policy experts have recommended HRAs for national programs like Medicare, and in 2014, the Veteran's Health Administration (VA) launched an online HRA. Yet little is known about how to effectively 
integrate HRAs into clinical practice to optimize patient engagement in recommended lifestyle changes like smoking cessation and weight management. One potential approach is to pair HRAs with health coaching. Health coaching is a collaborative model that integrates theories of human development and adult learning, in which a health coach works with patients to jointly set goals and action plans to maximize health and wellbeing. ${ }^{11}$ There is growing evidence that health coaching can improve health outcomes. ${ }^{12,13}$ Coupling an HRA with supportive health coaching may improve patient activation to engage in prevention programs as a strategy to reduce modifiable risk of cardiovascular disease. To this end, the purpose of this study was to assess the effectiveness of an HRA coupled with a brief health coaching intervention to activate veterans to enroll and participate in prevention programs chosen based on their needs and preferences.

\section{METHODS}

In brief, our primary objective was to assess effectiveness of an HRA linked with brief telephone-delivered health coaching compared to HRA alone in a pragmatic effectivenessimplementation Hybrid Type 1 randomized trial. ${ }^{14}$ The secondary objective was to provide information about processes necessary to implement such a program within a healthcare system. The protocol was approved by the VA's Central IRB. All eligible patients provided written informed consent prior to enrollment. Further study details can be found elsewhere. ${ }^{15}$

\section{Setting}

This study was conducted in three VHA primary care clinics in Ann Arbor, Michigan, Durham, North Carolina, and Greenville, North Carolina. Both Ann Arbor and Durham VA Medical Centers are large, academically affiliated medical centers, and the Greenville site is an affiliate of the Durham VA. The primary care population served by these facilities is approximately 19,000 for Ann Arbor, 22,000 for Durham, and 13,000 for Greenville.

\section{Participants}

Potentially eligible patients were identified through electronic medical records with eligibility criteria verified by telephone screening and baseline interviews. To be included in the study, patients had to be enrolled in VA primary care at that site and have one or more of the following risk factors: $\mathrm{BMI} \geq 30$, current smoker, and/or $<150$ min of moderate/vigorous physical activity per week. Based on electronic medical records, telephone screenings, or baseline interviews, patients were excluded from participation for the following reasons: dementia or other memory loss condition, active diagnosis of psychosis, serious personality disorder, current uncontrolled substance abuse disorder, severely impaired hearing or speech, unable to speak English, serious/terminal illness as indicated by referral to hospice or palliative care, no access to a telephone, nursing home resident, or participating in a prevention program or another VA study within the last 6 months.

\section{Randomization and Allocation Concealment}

Following completion of baseline visit (including completion of informed consent and all baseline measures), patients were randomized in a 1:1 ratio to either the HRA + coaching intervention or HRA only. The randomization scheme was created by a statistician prior to study start and included a block size of four and was stratified by region (Michigan and North Carolina). Research assistants were blinded to block size and did not have access to the group assignment until after participants completed their baseline survey and outcome assessments. For outcome assessment, 1 month calls were conducted by research assistants from alternate sites (e.g., Durham's research assistant called Ann Arbor participants). Six-month outcome assessments were conducted on-site, because repeat lab studies were needed. In general, these were conducted by the same research assistant who enrolled the patient meaning that it was possible that they remembered participants' group assignment from 6 months ago.

\section{Health Risk Assessment}

All participants completed the VA's online HRA. The HRA was developed by the VA National Center for Health Promotion and Disease Prevention and launched nationally through the patient web-portal, MyHealtheVet, in September 2014. This output included a calculation of "health age," which was based on risk modeling. The HRA user's health age could show a value higher or lower than their chronological age based on lifestyle choices, family risk, and biological values. Participants also had access to detailed information for each health topic. The HRA is publicly available and can be accessed online after free registration (www.myhealth.va.gov/mhv-portal-web/web/myhealthevet/ healtheliving-assessment (Accessed Feb 6, 2018)).

\section{Intervention}

As with many published effective health coaching interventions, coaching within this trial utilized motivational interviewing techniques. ${ }^{16}$ Coaches worked with participants to co-develop a goal to reduce their modifiable CVD risk by choosing a prevention program to enroll in that was aligned with the participant's own values and preferences and that addressed their chosen risk factor (e.g., smoking) from their personalized list, displayed within their HRA report. Coaches used HRA-generated health summary outputs to facilitate patients' understanding of their current risks and how much that risk could be reduced by improved diet, increased physical activity, losing weight, and/or quitting tobacco use. Program choices included both VA and community-based structured prevention programs. 
Patients were randomly assigned to one of two health coaches. There were two planned coaching telephone calls: the first occurred within 1 week of baseline surveys and the second 1 month later. Patients had the same health coach for both calls. The first call followed a semi-scripted approach that included the following steps: (1) review of HRA results focusing on the difference between modifiable and nonmodifiable risk; (2) assessment of patient preferences and values related to modifiable risk factors; (3) prioritization of approaches to impact modifiable risks; (4) helping patients choose a prevention program that would best meet their needs and preferences; (5) collaboratively developing a SMART (small, measureable, attainable, relevant, and timely) goal aimed at enrolling in the chosen prevention program to address modifiable risk factor; and (6) assessing readiness and confidence to take the final steps needed to enroll and participate in that program. The patient's prevention goal was documented in a "Prevention Action Plan" that was mailed to the participant and included as a note in the electronic medical record.

One month after the initial coaching call, each patient received a follow-up coaching call from their health coach. The purpose of this call was to review progress toward completing their goal to engage in a structured prevention program. If the participant enrolled in their chosen prevention program, the coach congratulated them and encouraged their continued participation. If the participant had not yet enrolled, or was encountering challenges, the coach problem-solved with the patient to help overcome those challenges and set a new SMART goal, which may have included a different program.

\section{Training and Supervision of Health Coaches}

Health coach training included didactic components, hands-on practice, and role play. We used three strategies to ensure high fidelity to coaching delivery as designed: (1) coaches used computer-assisted semi-structured scripts to guide each coach call; (2) all telephone coaching sessions were audio-recorded, and a sample of recordings were scored and used to augment feedback to coaches throughout the study; and (3) supervision sessions were conducted weekly for the first 6 months, then every 2 weeks, in which coaches presented challenging calls and skills were practiced. The study co-leaders (EO and LD) independently reviewed approximately $10 \%$ of coaching calls using a checklist of elements deemed essential to the call. Those findings were discussed with the coaches' during regular supervision sessions led by a study co-investigator (JMG).

\section{Usual Care}

Usual care patients received a printed copy of their HRA output and were encouraged to discuss any questions they had with their primary care team. The primary care team was also alerted to their patients' participation in the study via a standard note in the electronic medical record.

\section{Outcomes}

The primary outcome was enrollment in a structured prevention program by 6 months. Participants were considered enrolled if they answered affirmatively to enrolling in a program and if they provided the name of the program at one or both of the 1- or 6-month assessments. Secondary outcomes included participation in a prevention program, change in scores on the Patient Activation Measure (PAM), and the Framingham Risk Score (FRS). Participants were considered to have participated in a prevention program if they were enrolled in that program and reported attending at least one session. The PAM is a 13item measure that assesses activation in terms of an individuals' knowledge, skills, beliefs, and confidence for managing their health. ${ }^{17}$ PAM scores show high construct validity in that they are highly correlated with patients' engagement in prevention services such as weight loss, smoking cessation, and willingness to exercise. ${ }^{18}$ Patients with higher PAM scores are also less likely to smoke, be overweight, and less likely to be hospitalized or visit the ED, and they correlate with physiologic parameters such as blood pressure and lipid levels. ${ }^{19,20}$ The FRS is a validated assessment of cardiovascular risk that has been used widely in research and clinical practice. ${ }^{21}$ In clinical trials of behavior interventions, the FRS has been shown to be responsive to change, including coaching interventions. ${ }^{12}$ Blood pressure and lipid measurements from blood tests were used to calculate the FRS at the baseline and 6-month visits. Prevention program enrollment, program participation, and PAM were assessed at baseline, 1 , and 6 months. The FRS was assessed at baseline and 6 months only to allow sufficient time for potential positive health changes to impact FRS components (e.g., blood pressure, lipids).

\section{Sample Size}

Sample size estimates were based on the primary hypothesis that a greater proportion of patients randomized to the health coaching intervention arm would enroll in prevention programs as compared to HRA only usual care patients. We anticipated approximately 5\% of usual care patients would enroll in a prevention program by 6 months. To detect a minimum of $8 \%$ difference in enrollment rates with $80 \%$ power and Type I error of 5\%, we needed to randomize 200 patients per arm.

\section{Statistical Analysis}

The primary outcome, cumulative enrollment in prevention programs by 6-month assessment, was evaluated via selfreport at months one and six. As defined by the eligibility criteria, all patients had a value of 0 at baseline. Logistic regression, with an indicator variable for the intervention arm effect and adjusted for region, was used to test the primary hypothesis of a higher enrollment rate attributed to the coaching intervention. 
For the secondary outcomes of PAM and FRS, we used a general linear model with unstructured covariance (PROC MIXED in SAS, Cary, NC). In the PAM model, parameters included a common intercept, indicator variables for 1 and 6 months, indicator variables for intervention arm interacted with time of assessment, and region. The FRS model only included the 6-month indicator variables. Contrast statements were used to estimate the difference between groups in mean PAM and FRS change from baseline and each follow-up. Finally, a logistic regression model similar to that for the primary outcome was used to estimate group differences in the secondary outcome of participation in a prevention program over the 6 months of follow-up.

All available data, including information from participants who subsequently discontinued the study, were used for analyses. The PAM and FRS secondary outcome analyses were conducted with full likelihood methods, which are valid under a missing data framework in which the missing values may depend on intervention group, region, and any observed outcome values. ${ }^{22}$ For the primary outcome of enrollment and secondary outcome of participation, we also conducted sensitivity analyses where missing 6-month outcomes were imputed with both possible outcomes (i.e., all missing enrolled or all missing not enrolled) to examine the range of possible effects under varying missing data assumptions.

\section{RESULTS}

\section{Study Participants}

Recruitment started in November 2014 and ended in May 2016. Of 7961 veterans who met initial electronic medical record eligibility criteria, 38\% $(n=3007)$ were sent a recruiting letter; $18 \%$ of those individuals were screened as eligible $(n=555)$. Of the 3007 who were identified by medical records, 1216 (46\%) were not contacted either, because recruitment ended or patients could not be reached by phone. Of patients who screened eligible by phone, $79 \%$ consented $(n=$ 436) of whom $96 \%(n=417)$ were randomly assigned to one of the two arms (Fig. 1).

Table 1 shows baseline demographic and clinical characteristics for study participants. On average, participants were 56 years old $(\mathrm{SD}=12.2)$. A large percentage of participants were African American (40\%), most were male (85\%), and over $25 \%$ of our participants reported inadequate income to cover bills at the end of the month reflecting a high proportion of financial vulnerability. In terms of health status, $69 \%$ of patients reported their general health as excellent, very good, or good.

Eighty percent of participants met enrollment criteria because of weight (BMI $\geq 30$ ), $50 \%$ for being moderately/ vigorously active for less than $150 \mathrm{~min} /$ week, and $39 \%$ were active smokers. Approximately half of the participants had more than one eligible risk factor. The mean HRA-generated health age was 60 years, while the participants' average chronological age was 56 primarily reflecting their excess cardiovascular risk (Table 1).

\section{Adherence to the intervention}

Of the 208 participants randomized to the intervention, 194 participants $(93 \%)$ completed the first coaching call, and 182 participants $(88 \%)$ completed the second; 14 participants $(7 \%)$ of intervention participants did not complete either call. The mean duration of the first coaching call was 34 min (SD $14 \mathrm{~min}$ ), and the mean duration of the second coaching call was $12 \mathrm{~min}$ (SD $10 \mathrm{~min}$ ).

\section{Outcomes}

At 6 months, intervention participants reported higher enrollment in a prevention program, 51 vs $29 \%(\mathrm{OR}=2.5 ; 95 \% \mathrm{CI}$ : $1.7,3.9, p<0.0001)$ and higher participation in a prevention program, 40 vs. $23 \%(\mathrm{OR}=2.3 ; 95 \% \mathrm{CI}: 1.5,3.6 ; p<.001)$ compared to HRA only usual care participants (Table 2). There was no difference in PAM scores between groups at the 1-month assessment. However, at 6 months after baseline, intervention participants showed greater improvement in PAM scores than usual care participants (mean difference $=2.5$; 95\% CI: $0.2,4.7 ; p=0.03$ ).

The FRS showed almost no change over time from baseline to 6 months for both groups (mean difference $=0.7 ; 95 \% \mathrm{CI}$ : $-0.7,2.2 ; p=0.33)$.

Results were robust to missing data assumptions; in our sensitivity analyses for the primary outcome of enrollment, the OR with 95\% CI ranged from $2.1(1.4,3.2)$ with all missing assumed to be not enrolled to $2.7(1.8,3.9)$ for all missing assumed to be enrolled.

\section{Prevention Programs Chosen by Intervention Participants}

We categorized prevention programs chosen by participants by type (diet/weight loss, exercise, or smoking cessation), and as VA-sponsored, or community-based. Among the $51 \%(n=$ 91 of 177) of intervention patients who endorsed enrolling in a prevention program by 6 months, $52 \%$ selected diet or weight loss programs, $26 \%$ selected exercise programs, and $19 \%$ selected smoking cessation (3\% remained uncharacterized). Overall, $55 \%$ of these participants selected VA programs and $45 \%$ selected non-VA programs (Table 3 ).

\section{DISCUSSION}

To our knowledge, this is the first trial to test an intervention that coupled HRA with a telephone-delivered health coaching intervention designed to help participants enroll in a structured prevention program. Health coaches were successful in engaging participants in a discussion about prevention, guiding them to a program that matched their goals, which resulted in intervention participants enrolling and participating in 


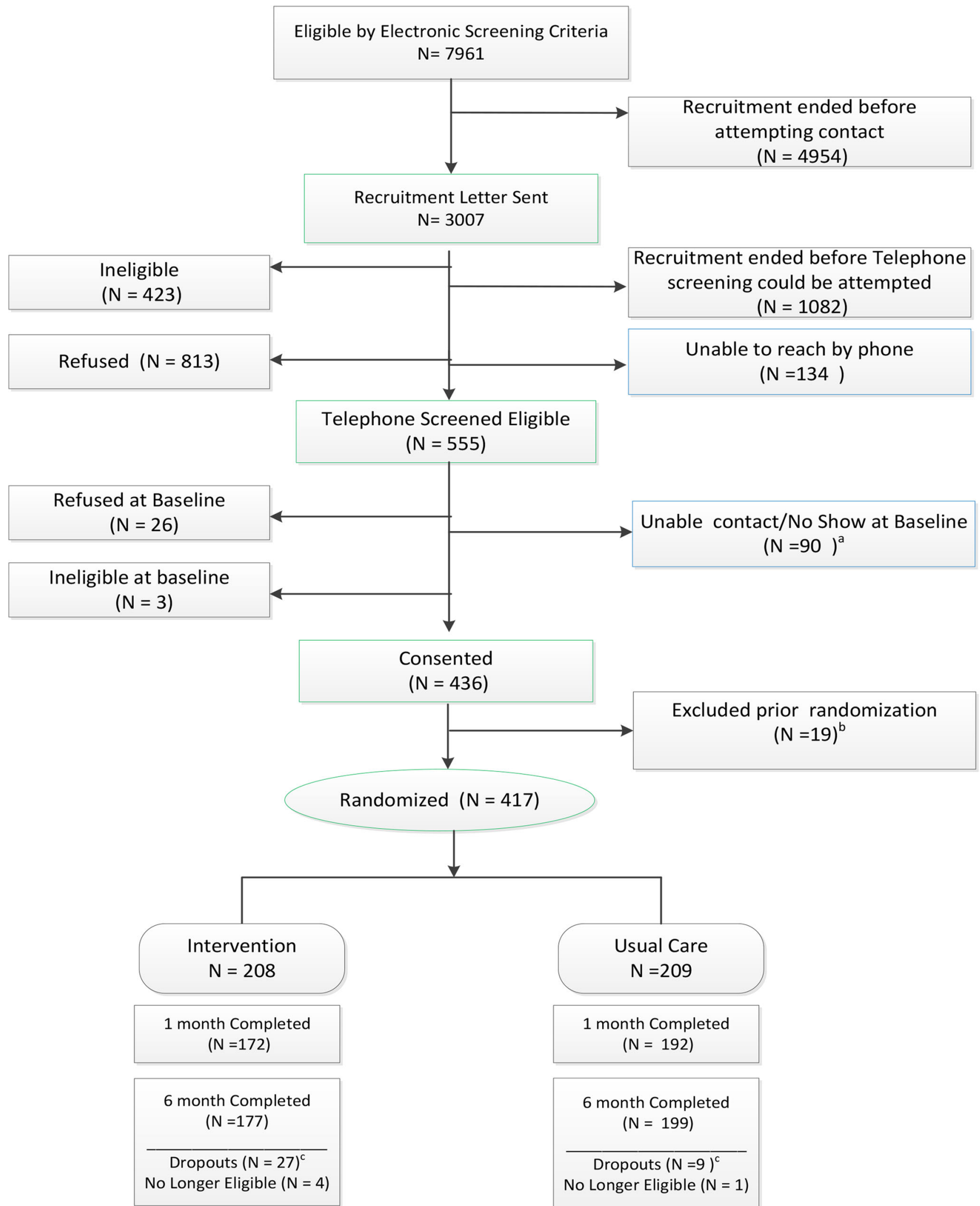

Foot Note: a- patients were unable to complete recruitment process due to phone outages, not showing for appointment, non-responders to phone calls. $b$ - includes formal and informal withdrawal prior to randomization as well as exclusion for failure to complete the health risk assessment. $\mathrm{c}$ - includes formal and informal withdrawal post randomization.

Figure 1 Consort diagram showing screening, enrollment, and follow-up. 
Table 1 Demographic and Clinical Characteristics of Study Participants

\begin{tabular}{|c|c|c|c|}
\hline & Overall $(n=417)$ & Intervention $(n=208)$ & Usual care $(n=209)$ \\
\hline Chronological age, mean (SD) & $55.8(12.2)$ & $55.3(12.7)$ & $56.3(11.7)$ \\
\hline Health risk assessment (HRA)-generated age, mean (SD) & $60.4(12.6)$ & $60.0(12.9)$ & $60.7(12.4)$ \\
\hline HRA age minus actual age, mean (SD) & $4.6(5.7)$ & $4.7(5.6)$ & $4.4(5.9)$ \\
\hline Male, $n(\%)$ & $356(85.4)$ & $172(82.7)$ & $184(88.0)$ \\
\hline \multicolumn{4}{|l|}{ Race, $n(\%)$} \\
\hline Black or African American & $169(40.5)$ & $90(43.3)$ & $79(37.8)$ \\
\hline White & $209(50.1)$ & $99(47.6)$ & $110(52.6)$ \\
\hline Other & $39(9.4)$ & $19(9.1)$ & $20(9.6)$ \\
\hline \multicolumn{4}{|l|}{ Ethnicity, $n(\%)$} \\
\hline Hispanic or Latino & $13(3.1)$ & $7(3.4)$ & $6(2.9)$ \\
\hline Married/living as married, $n(\%)$ & $213(51.1)$ & $96(46.2)$ & $117(56.0)$ \\
\hline At least high school education, $n(\%)$ & $410(98.3)$ & $206(99.0)$ & $204(97.6)$ \\
\hline Employed full/part time, $n(\%)$ & $153(36.7)$ & $79(38.0)$ & $74(35.4)$ \\
\hline Inadequate income, $n(\%)$ & $111(26.6)$ & $57(27.4)$ & $54(25.8)$ \\
\hline Hypertension, $n(\%)$ & $257(61.6)$ & $129(62.0)$ & $128(61.2)$ \\
\hline Diabetes, $n(\%)$ & $116(27.8)$ & $54(26.0)$ & $62(29.7)$ \\
\hline General health, excellent/very good/good, $n(\%)$ & $287(68.8)$ & $141(67.8)$ & $146(69.9)$ \\
\hline \multicolumn{4}{|l|}{ Inclusion criteria } \\
\hline Current smoker of cigarettes or other tobacco, $n(\%)$ & $163(39.1)$ & $88(42.3)$ & $75(35.9)$ \\
\hline $\mathrm{BMI} \geq 30, n(\%)$ & $332(79.6)$ & $161(77.4)$ & $171(81.8)$ \\
\hline Physical activity $<150$ min per week, $n(\%)$ & $209(50.1)$ & $99(47.6)$ & $110(52.6)$ \\
\hline \multicolumn{4}{|l|}{ No. of inclusion criteria met } \\
\hline 1 & $174(41.7)$ & $88(42.3)$ & $86(41.1)$ \\
\hline 2 & $199(47.7)$ & $100(48.1)$ & $99(47.4)$ \\
\hline 3 & $44(10.6)$ & $20(9.6)$ & $24(11.5)$ \\
\hline
\end{tabular}

*Responded yes to "Must cut back on things to pay bills or have difficulty paying bills at the end of the month"

programs to a significantly greater degree than participants not receiving coaching. Intervention participants also showed significant improvement in PAM scores, a measure that captures patients' knowledge, skills, and confidence in management of their health. In other studies, higher PAM scores correlate with patients who are more engaged in prevention and more likely to exercise. ${ }^{23}$ The 2.5 point change in PAM scores at 6 months translates into an effect size (Cohen's D) of 0.19 which is small and may not be clinically meaningful. In this short duration study, the intervention did not, however, improve overall cardiovascular risk as measured by the FRS.

The degree of prevention program enrollment observed in this study (over half enrolled, and $40 \%$ participating by 6 months) is much higher than reported in other studies that seek to engage primary care patients in prevention programs.
For example, only $10 \%$ of veterans eligible for the VA's national weight management program ever enroll. ${ }^{24}$ In part, this may be explained by integrating results from an HRA with health coaching. The health coaches guided patients to first understand their risk, the portion of it that was modifiable, and what they could do to improve their risk. They emphasized the difference between their current health state and their ideal health state, and linked that concept to participants' values and hopes for the future. Rather than coaching to change a specific health behavior selected by the participant, coaches helped participants set a specific goal to enroll in a structured program that best matched their circumstances and preferences. In this way, the intervention was able to address an array of risky behaviors (e.g., weight, tobacco use) with a focus on helping to motivate patients to engage in effective, structured

Table 2 Main Outcomes: Model-Estimated Changes in Enrollment and Participation in Prevention Program, Patient Activation, and Framingham Risk Score

\begin{tabular}{|c|c|c|c|c|}
\hline & Intervention $N=\mathbf{2 0 8}$ & Usual care $N=209$ & $\begin{array}{l}\text { Odds ratio or mean } \\
\text { difference }(95 \% \text { CI) }\end{array}$ & $P$ value \\
\hline \multicolumn{5}{|c|}{ Enrollment in prevention program percent, $(95 \% \mathrm{CI})$} \\
\hline Ever enrolled by 6 months & $51 \%(43 \%, 58 \%)$ & $29 \%(23 \%, 36 \%)$ & $2.5(1.7,3.9)$ & $<.0001$ \\
\hline Ever participated by 6 months & $40 \%(33 \%, 48 \%)$ & $23 \%(17 \%, 29 \%)$ & $2.3(1.5,3.6)$ & 0.0004 \\
\hline \multicolumn{5}{|c|}{ Patient activation measure score ${ }^{+}$, mean, $(95 \%$ CI $)$} \\
\hline Baseline & $61.5(60.3,62.7)$ & $61.5(60.3,62.7)$ & & \\
\hline 1 month & $63.2(61.5,64.9)$ & $61.8(60.1,63.4)$ & $1.5(-0.8,3.7)$ & 0.20 \\
\hline 6 months & $66.3(64.6,68.1)$ & $63.9(62.2,65.5)$ & $2.5(0.2,4.7)$ & 0.03 \\
\hline \multicolumn{5}{|c|}{ Framingham risk score, mean $(95 \% \mathrm{CI})$} \\
\hline Baseline & $22.3(20.7,24.0)$ & $22.3(20.7,24.0)$ & & \\
\hline 6 months & $22.7(20.8,24.5)$ & $21.9(20.1,23.7)$ & $0.7(-0.7,2.2)$ & 0.33 \\
\hline
\end{tabular}

Values shown in table reflect estimates derived from logistic regression (enrollment and participation) and general linear models (PAM, Framingham Risk). Note that the baseline-estimated group means are constrained to be equal

${ }^{+}$Range (0-100) 
Table 3 Breakdown of Program Choice for Enrolled Intervention Participants $(n=91)$

\begin{tabular}{|c|c|c|c|c|c|c|}
\hline & \multicolumn{2}{|l|}{ Total } & \multicolumn{2}{|l|}{ VA program } & \multicolumn{2}{|c|}{ Non-VA program } \\
\hline & Number & Percentage & Number (\%) & Percentage & Number & Percentage \\
\hline Diet/weight loss & 47 & 52 & 38 & 42 & 9 & 10 \\
\hline Exercise & 24 & 26 & 3 & 3 & 21 & 23 \\
\hline Smoking cessation & 17 & 19 & 9 & 10 & 8 & 9 \\
\hline Other program & 3 & 3 & & 0 & 3 & 3 \\
\hline Total & 91 & 100 & 50 & 55 & 41 & 45 \\
\hline
\end{tabular}

prevention programs. This approach leverages alreadyavailable prevention programs and requires less time than would be necessary to address changing the behavior itself. By focusing on three highly prevalent risk factors (obesity, physical inactivity, and smoking), the intervention was able to cover a broad range of high-risk patients. Additionally, the intervention has great potential for adoption within clinical settings, because it is a relatively low-resource approach. Furthermore, this approach may increase reach, because many patients prefer telephone coaching for its convenience and personal approach. ${ }^{25,26}$

A strength of our study is that we designed and conducted the intervention using elements that are widely available in VA and other large healthcare systems (e.g., online HRA, telehealth coaches). VA and other health systems are working to understand how to best incorporate health risk assessment into routine primary care as well as encouraging effective prevention programs for their patients. A recent trial of an HRA, conducted in 18 primary care practices showed that patients who used a comprehensive HRA, had higher screening rates for health behaviors and mental health risks, and were more likely to set specific health care goals (e.g., lose weight) compared to controls. ${ }^{27}$ Patients who received the HRA in that study also reported feeling their clinicians cared more and showed more concern for their issues. Our current study extends these findings to show that when coupled with brief health coaching, patients are more likely to enroll and participate in prevention programs compared to using an HRA alone, thereby taking patients beyond goal setting to action.

\section{Limitations}

This study has several limitations. First, it was conducted in three VA facilities. Resources, primary care practices, and patient populations in the VA are different from non-VA settings. For example, our study participants were mostly men with a higher proportion of financial instability and a higher percentage of African Americans than most non-VA settings. Second, our primary outcome, enrollment in a prevention program, was determined by patient self-report. We chose this method, because we knew that some patients would prefer to join non-VA prevention programs, and in fact, $45 \%$ of participants chose community-based prevention programs which do not typically have mechanisms to share enrollment and participation rates. We did, however, conduct a VA medical record review to determine if patients who reported selecting a VA weight management program showed evidence of attending. We found medical record evidence of enrollment (at least one visit) for 34 of the 38 patients who endorsed enrolling in a VA weight management program (89\%); only 13 of 331 patients who did not endorse enrollment showed evidence of a visit $(4 \%)$, (kappa $=0.77)$ which is a high level of agreement. A third limitation is that research assistants may not have been blinded at the 6-month outcome assessments. Lastly, while the intervention did improve enrollment in prevention programs, there was no improvement in cardiovascular risk as measured by the FRS. One explanation for this is that the short duration of this study (6 months) was not long enough for patients to complete their chosen program, sustain their behavior changes, and experience the longer-term benefits in improved CVD risk as computed using the FRS. Because the intervention was designed to improve prevention program enrollment and not the specific behavior itself (e.g., smoking cessation), we did not track specific risk factor behavior change.

\section{CONCLUSION}

Among veterans enrolled in primary care, completing a comprehensive HRA coupled with brief telephone health coaching increases patient activation and subsequent enrollment and participation in structured prevention programs. Next steps should concentrate on how best to incorporate this relatively low-resource intensive intervention into routine primary care practice.

Acknowledgments: This project was funded by the Department of Veterans Affairs, Health Services Research and Development Service (CRE 12-288). We are also grateful to the leadership and staff of the VA's National Center Health Promotion and Disease Prevention (NCP) for the constant support throughout this project, including Dr. Jane Kim, Chief Consultant (NCP), and Ms. Kathleen Pitman. We also acknowledge the dedication and professionalism of our two health coaches, Ms. Karen Juntilla and Ms. Courtney White-Clark.

Corresponding Author: Eugene Z. Oddone, MD, MHSc; Center for Health Services Research in Primary Care Durham VA Medical Center, Durham, NC, USA (e-mail: eugene.oddone@va.gov).

\section{Compliance with ethical standards:}

Conflict of interest: The authors declare that they do not have a conflict of interest. 


\section{REFERENCES}

1. Patel SA, Winkel M, Ali M, Narayan V, Mehta NK. Cardiovascular mortality associated with 5 leading risk factors: national and state preventable fractions estimated from survey data. Ann Intern Med 2015;163:245-53.

2. Danaei G, Ding EL, Mozaffarian D, Taylor B, Rehm J, Murray CJ, et al. The preventable causes of death in the United States: comparative risk assessments of dietary, lifestyle and metabolic risk factors. PloS Med 2009;6(4):e10000058

3. Nolte E, McKee M. Variations in amenable mortality-Trends in 16 highincome nations. Health Policy 2011;103:47-52.

4. Fryar CK, Herrick K, Afful J, Odgen C. Cardiovascular disease risk factors among male veterans, U.S., 2009-20012. Am J Prev Med 2016;50:101-105.

5. IOM. Returning Home from Iraq and Afghanistan: Preliminary Assessment of Readjustment Needs of Veterans, Service Members, and Their Families. Washington, DC:: The National Academies Press; 2010.

6. Haibach JP, Haibach MA, Hall KS, Masheb RM, Little MA, Shepardson RL, et al. Military and veteran health behavior research and practice: challenges and opportunities. J Behav Med 2017;40:175-193.

7. Kinsinger LS. Disease Prevention in the Veterans Health Administration. NC Med J 2015;76(5):335-8.

8. Sheridan SL, Viera AJ, Krantz MJ, Ice CL, Steinman LE, Peters KE, et al. The effect of giving global coronary risk information to adults. Acrh Intern Med 2010;170(3);230-39.

9. Loeppke R, Edington DW, Beg S. Impact of prevention plan on employee health risk reduction. Population Health Management 2010;13:275-84

10. Powers B, Dannus S, Gruber J, Olsen MK, Oddone EZ, Bosworth HB. The effectiveness of personalized coronary heart and stroke risk communication. Amer Heart $J$ 2011;161:673-80

11. Wolever RQ, Eisenberg DM. What is health coaching anyway? Arch Intern Med 2011;171:2017-18.

12. Edelman D, Oddone EZ, Liebowitz RS, Yancy WS, Olsen MK, Jeffreys AS, et al. A multidimensional integrative medicine intervention to improve cardiovascular risk. J Gen Intern Med 2006; 21(7):728-34.

13. Eakin EG, Lawler SP, Vandelanotte C, Owen N. Telephone interventions for physical activity and dietary behavior change: a systematic review. Am J Prev Med. 2007;32:419-434.

14. Curran, GM, Bauer M, Mittman B, Pyne JM, Stetler C. Effectivenessimplementation hybrid designs: combining elements of clinical effectiveness and implementation research to enhance public health impact. Medical Care 2012; 50(3), 217-226
15. Oddone EZ, Damschroder LJ, Geirisch J, Olsen M, Fagerlin A, Sanders $\mathbf{L}$, et al. A coaching by telephone intervention for veterans and care team engagement (ACTIVATE): a study protocol for a hybrid type 1 effectivensess-implementation randomized controlled trial. Contemp Clin Trials 2017;55:1-9.

16. Sontag U, Wiesner J, Fahrenkrog S, Renneberg B, Braun V, Heintze C. Motivational interviewing and shared decision making in primary care. Pat Educ and Counsel 2011; doi https://doi.org/10.1016/j.pec.2011.07. 026

17. Hibbard JH, Mahoney ER, Stockard J, Tusler M. Development and testing of a short form of the patient activation measure. Health Services Research 2005;40:1918-1930.

18. Hibbard JH, Mahoney E R, Stock R, Tusler M. Do increases in patient activation result in improved self-management behaviors? Health Services Research 2007;42:1443-1463.

19. Skolasky RL, Green AR, Scharfstein D, Boult C, Reider L, Wegener ST. Psychometric properties of the patient activation measure among multimorbid older adults. Health Services Research 2011;46:457-478.

20. Remmers C, Hibbard J, Mosen DM, et al. Is patient activation associated with future health outcomes and healthcare utilization among patients with diabetes? J Ambulatory Care Manage 2009;32:320-327.

21. D'Agostino RB Sr, Vasan RS, Pencina MJ, et al. General cardiovascular risk profile for use in primary care: the Framingham Heart Study. Circulation 2008; 117:743-53.

22. Olsen MK, Stechuchak KM, Edinger JD, Ulmer CS, Woolson RF. Move over LOCF: principled methods for handling missing data in sleep disorder trials. Sleep Med. 2012;13:123-32.

23. Greene J, Hibbard JH. Why does patient activation matter? An examination of the relationships between patient activation and healthrelated outcomes. J Gen Intern Med 2012;27:520-526.

24. Kahwati LC, Lance TX, Jones KR, Kinsinger LS. RE-AIM evaluation of the veterans health administration's MOVE! Weight management program. TBM 2011;1:551-60.

25. Bosworth HB, Olsen MK, Grubber JM, et al. Two self-management interventions to improve hypertension control: a randomized trial. Ann Intern Med 2009;151:687-695

26. Lutes LK, Damschroder LJ, Masheb R, Kim HM, Gillon L, Holleman RG, et al. Behavioral treatment for veterans with obesity: 24-month weight outcomes from the ASPIRE-VA small changes randomized trial. J Gen Intern Med 2017;32:S40-S47.

27. Krist AH, Glasgow RE, Heurtin-Roberts S, Sabo RT, Roby DH, Sheinfeld-Gorin SN, et al. The impact of behavioral and mental health risk assessments on goal setting in primary care. TBM 2016;6:212-219. 